\title{
Cycle relations on Jacobian varieties
}

\author{
Gerard van der Geer and Alexis Kouvidakis \\ (with an appendix by Don Zagier)
}

\begin{abstract}
Dedicated to Jozef Steenbrink on the occasion of his 60th birthday
\end{abstract}
\begin{abstract}
By using the Grothendieck-Riemann-Roch theorem we derive cycle relations modulo algebraic equivalence in the Jacobian of a curve. The relations generalize the relations found by Colombo and van Geemen and are analogous to but simpler than the relations recently found by Herbaut. In an appendix by Zagier, it is shown that these sets of relations are equivalent.
\end{abstract}

\section{Introduction}

Beauville showed in [Bea86] that the Chow ring with rational coefficients of an abelian variety possesses a double grading $C H_{\mathbb{Q}}(X)=\bigoplus C H_{(j)}^{i}(X)$ where $i$ refers to the codimension and $j$ refers to the action of the integers: $C H_{(j)}^{i}(X)=\left\{x \in C H^{i}(X): k^{*}(x)=k^{2 i-j} x\right\}$. The quotient $A(X)$ of the Chow ring modulo algebraic equivalence inherits this double grading $A(X)=\bigoplus A_{(j)}^{i}(X)$ and carries two multiplication laws, the intersection product $x \cdot y$ and the Pontryagin product $x * y$.

If $X=\operatorname{Jac}(C)$ is the Jacobian of a curve $C$ of genus $g$, then we can decompose the class $[C]$ of the image of the Abel-Jacobi map of $C$ as $[C]=\sum_{j=0}^{g-1} C_{(j)}$ with $C_{(j)} \in A_{(j)}^{g-1}(\operatorname{Jac}(C))$. Colombo and van Geemen proved (cf. [CV93]) that for a curve $C$ with a map of degree $d$ to $\mathbf{P}^{1}$, the component $C_{(j)}$ vanishes for $j \geqslant d-1$. In [Her07] Herbaut extended this result and found cycle relations for curves having a $g_{d}^{r}$, i.e. a linear system of degree $d$ and projective dimension $r$, with $r \geqslant 2$.

It is the purpose of this note to show that one can use the Grothendieck-Riemann-Roch theorem to derive, in an easy way, the Colombo-van Geemen result as well as simple relations of higher degree.

Let $C$ be a smooth projective curve of genus $g$ over an algebraically closed field $K$.

Theorem 1.1. If $C$ has a base-point-free linear system $g_{d}^{r}$, then

$$
\sum_{a_{1}+\cdots+a_{r}=N}\left(a_{1}+1\right) ! \cdots\left(a_{r}+1\right) ! C_{\left(a_{1}\right)} * \cdots * C_{\left(a_{r}\right)}=0
$$

for every $N \geqslant d-2 r+1$.

For $N=d-2 r+1$, this relation coincides with Herbaut's relation (cf. [Her07, Theorems 1 and 8]). For higher values of $N$ they are in general different, but in an appendix we present a proof by Don Zagier that shows that Theorem 1.1 and Herbaut's Theorem 1 are equivalent.

Received 12 September 2006, accepted in final form 27 November 2006. 2000 Mathematics Subject Classification 14C25, $14 \mathrm{H} 40$.

Keywords: Jacobian variety, algebraic cycle.

This journal is (C) Foundation Compositio Mathematica 2007. 


\section{CyCle RELATIONS ON JACOBIAN VARIETIES}

\section{Preliminaries}

Let $C$ be a smooth projective curve of genus $g$ over an algebraically closed field $K$. We suppose that the curve $C$ has a base-point-free linear system $g_{d}^{r}$ of degree $d$ and projective dimension $r$. This defines a morphism $\gamma: C \rightarrow \mathbf{P}^{r}$. Let $J=\operatorname{Jac}(C)$ be the Jacobian of $C$.

We consider the incidence variety $Y \subset C \times \hat{\mathbf{P}}^{r}$ defined by

$$
Y=\left\{(p, \eta) \in C \times \hat{\mathbf{P}}^{r}: \gamma(p) \in \eta\right\},
$$

where $\hat{\mathbf{P}}^{r}$ is the dual projective space of $\mathbf{P}^{r}$. It has dimension $r$ and possesses the two projections $\tilde{\phi}$ and $\tilde{\alpha}$ onto $C$ and $\hat{\mathbf{P}}^{r}$. Note that $\tilde{\alpha}$ is finite of degree $d$ and $\tilde{\phi}$ is a $\mathbf{P}^{r-1}$-fibration. We shall write $\mathbf{P}^{r}$ for $\hat{\mathbf{P}}^{r}$.

We have the following diagram of morphisms

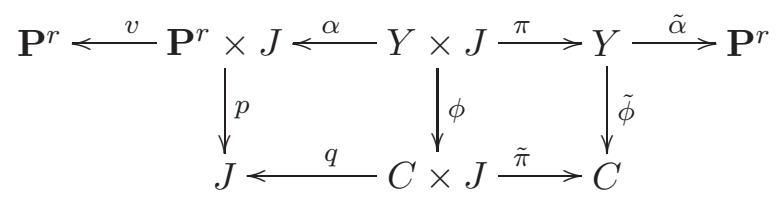

where the morphisms $v, p, q, \tilde{\pi}$ and $\pi$ are projections, and $\alpha=\tilde{\alpha} \times \operatorname{id}_{J}$ and $\phi=\tilde{\phi} \times \operatorname{id}_{J}$.

Let $P$ be the Poincaré bundle on $C \times J$ and set $L:=\phi^{*} P$, a line bundle on $Y \times J$. Put $\ell:=c_{1}(L)$ and $\Pi:=c_{1}(P)$.

The Chow ring of $\mathbf{P}^{r} \times J$ is generated over $C H^{*}(J)$ by the class $\xi=v^{*} h$ with $h$ a hyperplane in $\mathbf{P}^{r}$ with $\xi^{r+1}=0$. For a class $\beta \in C H^{*}\left(\mathbf{P}^{r} \times J\right)$ we have the relation (cf. [Ful98, Theorem 3.3, p. 64])

$$
\beta=\sum_{i=0}^{r} \beta_{i} \xi^{r-i} \quad \text { with } \beta_{i}=p_{*}\left(\beta \cdot \xi^{i}\right),
$$

where, by abuse of notation, we write here and hereafter $\beta_{i}$ for $p^{*}\left(\beta_{i}\right)$.

We let $x=\alpha^{*}(\xi)$ be the pull back of $\xi$. We let $\rho=\pi^{*} \tilde{\phi}^{*}$ (point) be the pull back class of a point on $C$. We work in the Chow ring up to algebraic equivalence. There we have the relations

$$
x^{r}=d \pi^{*}(\text { point }), \quad x^{r+1}=0, \quad \rho^{2}=0, \quad x^{r-1} \rho=\pi^{*}(\text { point }) .
$$

Recall the Fourier transform $F: A(X) \rightarrow A(X)$ for a principally polarized abelian variety $(X, \theta)$ of dimension $g$ (cf. [Bea86, Bea04]). It has the properties (i) $F \circ F=(-1)^{g}(-1)^{*}$, (ii) $F(x * y)=$ $F(x) \cdot F(y)$ and $F(x \cdot y)=(-1)^{g} F(x) * F(y)$, (iii) $F\left(A_{(j)}^{i}(X)\right)=A_{(j)}^{g-i+j}(X)$.

We have the relation $q_{*}\left(e^{\Pi}\right)=F[C]$ (cf. [Bea04, §3]). Comparing terms gives that $F\left[C_{(j)}\right]=$ $(1 /(j+2) !) q_{*}\left(\Pi^{j+2}\right)$ for $j=0, \ldots, g-1$. Note, also, that $q_{*} 1=q_{*} \Pi=0$. More generally, extending scalars to $\mathbb{Q}$ we have the relation

$$
q_{*}\left(e^{k \Pi}\right)=k^{2 g} F\left[\left(k^{-1}\right)^{*} C\right] \quad \text { for } k \in \mathbb{Z}_{\geqslant 1} .
$$

In fact, writing $[C]=\sum_{j=0}^{g-1} C_{(j)}$ we have $\left(k^{-1}\right)^{*}[C]=\sum_{j} k^{j+2-2 g} C_{(j)}$, hence

$$
k^{2 g} F\left[\left(k^{-1}\right)^{*} C\right]=F\left[\sum_{j} k^{j+2} C_{(j)}\right]=\sum_{j} k^{j+2} q_{*}\left(\Pi^{j+2} /(j+2) !\right)=q_{*}\left(e^{k \Pi}\right) .
$$




\section{G. VAN DER GeER AND A. KOUvidAKIS}

\section{The proof}

We shall prove that if $C$ has a base-point-free linear system $g_{d}^{r}$, then

$$
\sum_{a_{1}+\cdots+a_{r}=N}\left(a_{1}+1\right) ! \cdots\left(a_{r}+1\right) ! F\left[C_{\left(a_{1}\right)}\right] \cdots F\left[C_{\left(a_{r}\right)}\right]=0
$$

for every $N \geqslant d-2 r+1$.

We are going to apply the Grothendieck-Riemann-Roch theorem to the morphism $\alpha$ and the line bundle $L$. For $k \geqslant 1$ we put $V_{k}:=\alpha_{*}\left(L^{\otimes k}\right)$. Since $\alpha$ is a finite morphism of degree $d$ this is a vector bundle of rank $d$ and we get

$$
\operatorname{ch}\left(V_{k}\right)=\operatorname{ch}\left(\alpha_{!} L^{\otimes k}\right)=\alpha_{*}\left(e^{k \ell} \operatorname{td}_{\alpha}\right),
$$

with $\operatorname{td}_{\alpha}$ the Todd class of the morphism $\alpha$.

The Todd class $\operatorname{td}_{\alpha}$ is algebraically equivalent to a class of the form $A(x)+B(x) \rho$. Here $A=$ $\sum_{j=0}^{r-1} a_{j} x^{j}$ and $B=\sum_{j=0}^{r-1} b_{j} x^{j}$ are polynomials in $x$ and $a_{0}=1$. In fact, $\operatorname{td}_{\alpha}$ is the pull back under $\pi$ of $\operatorname{td}_{\tilde{\alpha}}$, an element of $A(Y)$. The ring $A(Y)$ is generated as an $A(C)$-module by $1, x_{1}, \ldots, x_{1}^{r-1}$ with $x_{1}=\tilde{\alpha}^{*}(h)$ and $A(C)$ is generated by 1 and the class of a point.

Proposition 3.1. For $k \in \mathbb{Z}_{\geqslant 1}$ we have in $A\left(\mathbf{P}^{r} \times J\right)$ the relation

$$
\operatorname{ch}\left(V_{k}\right)=d A(\xi)+\xi B(\xi)+k^{2 g} F\left[\left(k^{-1}\right)^{*} C\right] \xi A(\xi) .
$$

In particular, all $\mathrm{ch}_{j}\left(V_{k}\right)$ are divisible by $\xi$ for $j \geqslant 1$.

Before we give the proof of Proposition 3.1 we state a corollary and a lemma.

Proposition 3.1 gives an expression of the Chern characters of the bundles $V_{k}$. We can express the Chern classes of the bundles $V_{k}$ of rank $d$ in terms of the Chern characters by using the well-known formula (cf. [Mac95, ch. I $\left.\left(2.10^{\prime}\right)\right]$ )

$$
1+c_{1}\left(V_{k}\right) t+\cdots+c_{d}\left(V_{k}\right) t^{d}=\exp \left(\sum_{j \geqslant 1}(-1)^{j-1}(j-1) ! \operatorname{ch}_{j}\left(V_{k}\right) t^{j}\right) .
$$

Formula (4) combined with Proposition 3.1 will give us the vanishing relations we are asking for. For example, applying these formulas for $r=1$ and $k=1$ immediately gives us the theorem of Colombo-van Geemen [CV93] as we now show.

Corollary 3.2. If $C$ has a $g_{d}^{1}$, then $C_{(j)}=0$ for $j \geqslant d-1$.

Proof. Put $V=V_{1}$. We see $\operatorname{ch}(V)=d+n \xi+F[C] \xi$ for some $n$ (actually $n=1-d-g$ ). Since $\operatorname{ch}_{j}(V)$ is divisible by $\xi$ for $j \geqslant 1$ and $\xi^{2}=0$, formula (4) becomes in this case

$$
1+c_{1}(V) t+\cdots+c_{d}(V) t^{d}=1+\operatorname{ch}_{1}(V) t-\operatorname{ch}_{2}(V) t^{2}+\cdots+(-1)^{j-1}(j-1) ! \operatorname{ch}_{j}(V) t^{j}+\cdots .
$$

Therefore, $\operatorname{ch}_{j}(V)$ vanishes for $j>d$. Hence, $F[C]$ has no terms of codimension $d$ or more. Since $F\left[C_{(j)}\right]$ is of codimension $j+1$, it follows that $C_{(j)}=0$ for all $j \geqslant d-1$.

Lemma 3.3. In $A\left(\mathbf{P}^{r} \times J\right)$ the following relations hold for $\nu \geqslant 0$ :

$$
\alpha_{*}\left(\ell^{\mu} \cdot x^{\nu}\right)=\left\{\begin{array}{ll}
q_{*}\left(\Pi^{\mu}\right) \xi^{\nu+1} & \mu>0, \\
d \xi^{\nu} & \mu=0
\end{array} \quad \text { and } \quad \alpha_{*}\left(\ell^{\mu} \cdot x^{\nu} \cdot \rho\right)= \begin{cases}0 & \mu>0, \\
\xi^{\nu+1} & \mu=0 .\end{cases}\right.
$$

Proof. For the first relation. By (2) the coefficient of $\xi^{r-j}$ is given by

$$
\begin{aligned}
p_{*}\left(\alpha_{*}\left(\ell^{\mu} x^{\nu}\right) \xi^{j}\right) & =p_{*}\left(\alpha_{*}\left(\ell^{\mu} x^{\nu} \alpha^{*}(\xi)^{j}\right)\right)=p_{*}\left(\alpha_{*}\left(\phi^{*}(\Pi)^{\mu} x^{\nu+j}\right)\right) \\
& =q_{*}\left(\phi_{*}\left(\phi^{*}(\Pi)^{\mu} x^{\nu+j}\right)\right)=q_{*}\left(\Pi^{\mu} \phi_{*}\left(x^{\nu+j}\right)\right) .
\end{aligned}
$$




\section{CyCle RELATIONS ON JACOBIAN VARIETIES}

If $\nu+j=r$, then $x^{r}$ is algebraically equivalent to $d$ times point $\times J$ and since $\Pi$ is algebraically equivalent to 0 on point $\times J$, we get that any term with $\mu>0$ and $\nu+j=r$ contributes 0 . The term with $\nu+j=r-1$ contributes $q_{*}\left(\Pi^{\mu} \phi_{*}\left(x^{r-1}\right)\right)=q_{*}\left(\Pi^{\mu}\right)$ since $\left.\phi_{*}\left(x^{r-1}\right)\right)=1_{C \times J}$. If $\nu+j<r-1$ or $\nu+j>r$, then we get $q_{*}\left(\Pi^{\mu} \phi_{*}\left(x^{\nu+j}\right)\right)=q_{*}\left(\Pi^{\mu} 0\right)=0$. Finally, if $\mu=0$, we use that $x=\alpha^{*} \xi$, hence $\alpha_{*}\left(x^{\nu}\right)=d \xi^{\nu}$.

For the second relation. Observe that $\ell^{\mu} \cdot \rho=0$, if $\mu \geqslant 1$. Indeed, $\ell=\phi^{*} \Pi, \rho=\phi^{*}($ point $\times J)$ and $\Pi \cdot($ point $\times J)=0$. When $\mu=0$, we have $\alpha_{*}\left(x^{\nu} \rho\right)=\xi^{\nu} \alpha_{*} \rho=\xi^{\nu+1}$.

We now give the proof of Proposition 3.1.

Proof of Proposition 3.1. We have $\alpha_{*}\left(\ell^{\mu} x^{\nu} \rho\right)=0$ for all $\mu \geqslant 1, \nu \geqslant 0$. So in the contributions $\alpha_{*}\left(e^{k \ell} \operatorname{td}_{\alpha}\right)$ we get contributions of the form $\alpha_{*}\left(e^{k \ell} A(x)\right)$ and $\alpha_{*}(\rho B(x))$ only. We have

$$
\begin{aligned}
\alpha_{*}\left(e^{k \ell} A(x)\right) & =\alpha_{*}(A(x))+\sum_{\mu \geqslant 1} \frac{k^{\mu}}{\mu !} \alpha_{*}\left(\ell^{\mu} A(x)\right)=d A(\xi)+\sum_{\mu \geqslant 1} \frac{k^{\mu}}{\mu !} q_{*} \Pi^{\mu} \xi A(\xi) \\
& =d A(\xi)+q_{*}\left(e^{k \Pi}\right) \xi A(\xi)=d A(\xi)+k^{2 g} F\left[\left(k^{-1}\right)^{*} C\right] \xi A(\xi) .
\end{aligned}
$$

On the other hand, $\alpha_{*}(\rho B(x))=\xi B(\xi)$.

By using the relation

$$
k^{2 g} F\left[\left(k^{-1}\right)^{*} C\right]=q_{*}\left(e^{k \Pi}\right)=\sum_{\mu \geqslant 0} \frac{k^{\mu+2}}{\mu+2 !} q_{*} \Pi^{\mu+2}=\sum_{\mu \geqslant 0} k^{\mu+2} F\left[C_{(\mu)}\right],
$$

we get the following corollary of Proposition 3.1.

Corollary 3.4. We put $a_{j}=b_{j}=0$ for every $j \geqslant r$. With $A=\sum_{j=0}^{r-1} a_{j} x^{j}$ where $a_{0}=1$ and $B=\sum_{j=0}^{r-1} b_{j} x^{j}$ we have for $j \geqslant 1, k \geqslant 1$ that

$$
\operatorname{ch}_{j}\left(V_{k}\right)=\left(d a_{j}+b_{j-1}\right) \xi^{j}+\sum_{m=1}^{j-1} a_{m-1} k^{j-m+1} F\left[C_{(j-m-1)}\right] \xi^{m} .
$$

With $j \geqslant 1$ we put

$$
\operatorname{ch}_{j}\left(V_{k}\right)=A_{1}(j) \xi^{1}+\cdots+A_{r}(j) \xi^{r},
$$

where $A_{m}(j)$ is of codimension $j-m$. Please note that $\operatorname{ch}_{j}\left(V_{k}\right)$ is divisible by $\xi$ for $j \geqslant 1$ by Proposition 3.1.

Remark 3.5. The coefficient $A_{m}(j)$ depends on $k$, but for simplicity of notation we do not involve the index $k$ in the notation.

Then, for every $j \geqslant 1$ and $1 \leqslant m \leqslant r$, we have

$$
A_{m}(j)= \begin{cases}d a_{j}+b_{j-1} & m=j \\ a_{m-1} k^{j-m+1} F\left[C_{(j-m-1)}\right] & m<j \\ 0 & m>j .\end{cases}
$$

Since $\operatorname{rank}\left(V_{k}\right)=d$, the coefficient of $t^{M+1}$ of the right-hand side of (4) must be zero for every $M \geqslant d$. Let us write

$$
F(t)=\sum_{j \geqslant 1}(-1)^{j-1}(j-1) ! \operatorname{ch}_{j}\left(V_{k}\right) t^{j}
$$




\section{G. VAn Der Geer And A. Kouvidakis}

Note that $F(t)^{i}=0$ for every $i \geqslant r+1$ because $\operatorname{ch}_{j}\left(V_{k}\right)$ is divisible by $\xi$ for $j \geqslant 1$. Therefore, the right-hand side of (4) is equal to $\sum_{i=0}^{r}(1 / i !) F(t)^{i}$. The coefficient of $t^{M+1}$ in the polynomial $F(t)^{i}$, for $i \geqslant 1$, is equal to

$$
(-1)^{M+1-i} \sum_{\alpha_{1}+\cdots+\alpha_{i}=M+1}\left(\alpha_{1}-1\right) ! \cdots\left(\alpha_{i}-1\right) ! \operatorname{ch}_{\alpha_{1}}\left(V_{k}\right) \cdots \operatorname{ch}_{\alpha_{i}}\left(V_{k}\right) .
$$

We denote the expression $\left(\alpha_{1}-1\right) ! \cdots\left(\alpha_{i}-1\right)$ ! by $\alpha\{1, i\}$. Then we have that

$$
\sum_{i=1}^{r} \frac{(-1)^{i}}{i !} \sum_{\alpha_{1}+\cdots+\alpha_{i}=M+1} \alpha\{1, i\} \operatorname{ch}_{\alpha_{1}}\left(V_{k}\right) \cdots \operatorname{ch}_{\alpha_{i}}\left(V_{k}\right)=0
$$

for $M \geqslant d$, and so

$$
\sum_{i=1}^{r} \frac{(-1)^{i}}{i !} \sum_{\alpha_{1}+\cdots+\alpha_{i}=M+1} \alpha\{1, i\}\left[\sum_{m_{1}=1}^{r} A_{m_{1}}\left(\alpha_{1}\right) \xi^{m_{1}}\right] \ldots\left[\sum_{m_{i}=1}^{r} A_{m_{i}}\left(\alpha_{i}\right) \xi^{m_{i}}\right]=0 .
$$

Now the left-hand side of this is easily seen to be equal to

$$
\sum_{m=1}^{r} \sum_{i=1}^{m} \frac{(-1)^{i}}{i !} \sum_{\alpha_{1}+\cdots+\alpha_{i}=M+1} \alpha\{1, i\} \sum_{m_{1}+\cdots+m_{i}=m}\left[A_{m_{1}}\left(\alpha_{1}\right) \cdots A_{m_{i}}\left(\alpha_{i}\right)\right] \xi^{m} .
$$

We therefore have, for every $m=1, \ldots, r$ and $M \geqslant d$, that

$$
\sum_{i=1}^{m} \frac{(-1)^{i}}{i !} \sum_{m_{1}+\cdots+m_{i}=m} \sum_{\alpha_{1}+\cdots+\alpha_{i}=M+1} \alpha\{1, i\} A_{m_{1}}\left(\alpha_{1}\right) \cdots A_{m_{i}}\left(\alpha_{i}\right)=0 .
$$

With $M \geqslant d$, the case $m=r$ gives the relation

$$
\sum_{i=1}^{r} \frac{(-1)^{i}}{i !} \sum_{m_{1}+\cdots+m_{i}=r} \sum_{\alpha_{1}+\cdots+\alpha_{i}=M+1} \alpha\{1, i\} A_{m_{1}}\left(\alpha_{1}\right) \cdots A_{m_{i}}\left(\alpha_{i}\right)=0 .
$$

If we write

$$
B_{M}(i)=\sum_{m_{1}+\cdots+m_{i}=r} \sum_{\alpha_{1}+\cdots+\alpha_{i}=M+1} \alpha\{1, i\} A_{m_{1}}\left(\alpha_{1}\right) \cdots A_{m_{i}}\left(\alpha_{i}\right),
$$

then this relation becomes $\sum_{i=1}^{r}\left((-1)^{i} / i !\right) B_{M}(i)=0$ for every $M \geqslant d$. We analyze the dependence on $k$.

Proposition 3.6. We write $\sum_{i=1}^{r}\left((-1)^{i} / i !\right) B_{M}(i)=\sum_{s} \Gamma_{s} k^{s}$ as a polynomial in $k$. With $M \geqslant d$ we have that $\Gamma_{s}=0$ for $s>M+1$ and

$$
\Gamma_{M+1}=\frac{(-1)^{r}}{r !} \sum_{a_{1}+\cdots+a_{r}=M-2 r+1}\left(a_{1}+1\right) ! \cdots\left(a_{r}+1\right) ! F\left[C_{a_{1}}\right] \cdots F\left[C_{a_{r}}\right] .
$$

Proof. If $A_{m_{1}}\left(\alpha_{1}\right) \cdots A_{m_{i}}\left(\alpha_{i}\right)$ contains a factor with $m_{j}>\alpha_{j}$, then it vanishes. Otherwise, since $A_{m_{j}}\left(\alpha_{j}\right)=a_{m_{j}-1} k^{\alpha_{j}-m_{j}+1} F\left[C_{\alpha_{j}-m_{j}-1}\right]$, except when $m_{j}=\alpha_{j}$, in which case $A_{\alpha_{j}}\left(\alpha_{j}\right)=d a_{\alpha_{j}}+$ $b_{\alpha_{j}-1}$, the power of $k$ contained in $A_{m_{1}}\left(\alpha_{1}\right) \cdots A_{m_{i}}\left(\alpha_{i}\right)$ is equal to $\left(\alpha_{1}+\cdots+\alpha_{i}\right)-\left(m_{1}+\cdots+m_{i}\right)+\nu=$ $M+1-r+\nu$, where $\nu$ is given by $\nu=\#\left\{m_{j} \neq \alpha j, j=1, \ldots, i\right\} \leqslant i$. Now if $i<r$ the above number is less than $M+1$. On the other hand, if $i=r$, then $m_{1}=\cdots=m_{r}=1\left(\right.$ since $m_{i} \geqslant 1$ ) and therefore

$$
B_{M}(r)=\sum_{\alpha_{1}+\cdots+\alpha_{r}=M+1} \alpha\{1, r\} A_{1}\left(\alpha_{1}\right) \cdots A_{1}\left(\alpha_{r}\right) .
$$

Again, if a term of the above sum contains a factor $A_{1}(1)$, then the power of $k$ contained in this term is less than $M+1$. On the other hand, the sum of the terms with no factor of the form $A_{1}(1)$ 
is

$$
B_{M}^{\prime}(r)=\sum_{\alpha_{\mu} \geqslant 2, \alpha_{1}+\cdots+\alpha_{r}=M+1} \alpha\{1, r\} A_{1}\left(\alpha_{1}\right) \cdots A_{1}\left(\alpha_{r}\right) .
$$

Since $\alpha_{\mu} \geqslant 2$ we have that $A_{1}\left(\alpha_{\mu}\right)=k^{\alpha_{\mu}} F\left[C_{\alpha_{\mu}-2}\right]$. Therefore,

$$
\begin{aligned}
B_{M}^{\prime}(r) & =k^{M+1} \sum_{\alpha_{\mu} \geqslant 2, \alpha_{1}+\cdots+\alpha_{r}=M+1} \alpha\{1, r\} F\left[C_{\alpha_{1}-2}\right] \cdots F\left[C_{\alpha_{r}-2}\right] \\
& =k^{M+1} \sum_{a_{\mu} \geqslant 0, a_{1}+\cdots+a_{r}=M-2 r+1}\left(a_{1}+1\right) ! \cdots\left(a_{r}+1\right) ! F\left[C_{a_{1}}\right] \cdots F\left[C_{a_{r}}\right] .
\end{aligned}
$$

We now prove Theorem 1.1.

Proof of Theorem 1.1. Since we are working with $\mathbb{Q}$-coefficients, we conclude that the coefficient of $k^{M+1}$ (which is the maximum degree of $k$ involved) must be zero for $M \geqslant d$, which is relation (3) with $N=M-2 r+1 \geqslant d-2 r+1$. By applying the Fourier transform Theorem 1.1 follows.

Remark 3.7. We finish with a few remarks. Note that the existence of a base-point-free $g_{d}^{r}$ implies the existence of a base-point-free $g_{d-1}^{r-1}$ for which Theorem 1.1 provides a set of relations. Furthermore, the statement of Theorem 1.1 remains true if one replaces $d$ by any larger integer $d^{\prime}$, although the curve need not possess a base-point-free $g_{d^{\prime}}^{r}$. For $r=1$ this reflects the fact that the vanishing of $C_{(a)}$ implies the vanishing of $C_{\left(a^{\prime}\right)}$ for all $a^{\prime}>a$, cf. [Mar05, Corollary 24].

\section{ACKNOWLEDGEMEnTs}

The authors would like to thank Don Zagier for providing a proof of the equivalence of the two sets of relations. The first author would like to thank the Department of Mathematics at Heraklion for the hospitality and the excellent working conditions during his stay in June-July 2006.

\section{Appendix. The equivalence of two sets of relations}

\section{Don Zagier}

The relation of Herbaut mentioned in the introduction [Her07, Theorem 1], has the same general form as (1), but with much more complicated coefficients, namely

$$
\sum_{a_{1}+\cdots+a_{r}=N} B_{d}\left(a_{1}, \ldots, a_{r}\right) C_{\left(a_{1}\right)} * \cdots * C_{\left(a_{r}\right)}=0
$$

for all $N \geqslant 0$ if the curve $C$ has a $g_{d}^{r}$, where

$$
B_{d}\left(a_{1}, \ldots, a_{r}\right)=\sum_{i_{1}, \ldots, i_{r} \geqslant 1}(-1)^{d-i_{1}-\cdots-i_{r}}\left(\begin{array}{c}
d \\
i_{1}+\cdots+i_{r}
\end{array}\right) i_{1}^{a_{1}+1} \cdots i_{r}^{a_{r}+1} .
$$

This equation is vacuous if $N \leqslant d-2 r$ (because then all $B_{d}\left(a_{1}, \ldots, a_{r}\right)$ vanish, as we will see in a moment and as Herbaut also points out) and reduces to (1) if $N=d-2 r+1$, but the two equations differ in general. In both theorems, since the existence of a $g_{d}^{r}$ trivially implies the existence of a $g_{d-r+s}^{s}$ for all $s \leqslant r$, we can add to the given sets of relations the corresponding relations with $(d, r)$ replaced by $(d-r+s, s)$ for all $1 \leqslant s \leqslant r$. In this appendix, we prove that these two extended sets of relations are equivalent. 


\section{G. VAn Der Geer And A. Kouvidakis}

From the identity

$$
\sum_{d=0}^{\infty}(-1)^{d-i}\left(\begin{array}{l}
d \\
i
\end{array}\right) u^{d}=\frac{u^{i}}{(1+u)^{i+1}}
$$

we obtain the formula

$$
\sum_{d=0}^{\infty} B_{d}\left(a_{1}, \ldots, a_{r}\right) u^{d}=\frac{1}{1+u} P_{a_{1}+2}(u) \cdots P_{a_{r}+2}(u),
$$

where $P_{n}(u)(n \geqslant 1)$ is the power series without constant term given by

$$
P_{n}(u):=\sum_{i=1}^{\infty} i^{n-1}\left(\frac{u}{1+u}\right)^{i} \text { in } \mathbb{Z}[[u]] .
$$

Lemma A.1. We have the following:

(i) $P_{n}(u)$ is a polynomial of degree $n$; more precisely, $P_{n}(u)$ has the form $\sum_{m=1}^{n}(m-1) ! \mathfrak{S}_{n}^{(m)} u^{m}$ where each $\mathfrak{S}_{n}^{(m)}$ is a positive integer;

(ii) $P_{n}(-1)=0$ for $n>1$;

(iii) for each $n \geqslant 1$, one has the Laurent series identity

$$
\left.\frac{(n-1) !}{[\log (1+x)]^{n}}=P_{n}\left(\frac{1}{x}\right)+\mathrm{O}(x) \text { in } \mathbb{Q}\left[x^{-1}, x\right]\right] .
$$

Example A.2. The first five polynomials $P_{n}(u)$ are $u, u+u^{2}, u+3 u^{2}+2 u^{3}, u+7 u^{2}+12 u^{3}+6 u^{4}$ and $u+15 u^{2}+50 u^{3}+60 u^{4}+24 u^{5}$. We have

$$
\frac{4 !}{[\log (1+x)]^{5}}=\frac{24}{x^{5}}+\frac{60}{x^{4}}+\frac{50}{x^{3}}+\frac{15}{x^{2}}+\frac{1}{x}+0-\frac{x}{252}+\frac{x^{2}}{504}-\frac{19 x^{3}}{30240}-\frac{x^{4}}{20160}+\frac{53 x^{5}}{147840}-\cdots .
$$

Proof. The easiest approach (as usual) is to use generating functions. We have

$$
\sum_{n=1}^{\infty} P_{n}(u) \frac{t^{n-1}}{(n-1) !}=\sum_{i=1}^{\infty}\left(\frac{u e^{t}}{1+u}\right)^{i}=\frac{u e^{t}}{1-u\left(e^{t}-1\right)}
$$

and, hence,

$$
\frac{1}{(n-1) !} \mathbf{C}_{u^{m}}\left[P_{n}(u)\right]=\mathbf{C}_{t^{n-1}}\left[e^{t}\left(e^{t}-1\right)^{m-1}\right] \quad(m, n \geqslant 1) .
$$

(Here $\mathbf{C}_{x^{i}}[\Phi(x)]$ denotes the coefficient of $x^{i}$ in a polynomial, power series or Laurent series $\Phi(x)$.) This clearly vanishes for $n<m$, showing that $P_{n}$ is a polynomial of degree $\leqslant n$. It also implies the explicit formula for $P_{n}$ given in Lemma A.1(i), with

$$
\mathfrak{S}_{n}^{(m)}=\frac{n !}{m !} \mathbf{C}_{t^{n}}\left[\left(e^{t}-1\right)^{m}\right]=\mathbf{C}_{x^{n-m}}\left[\prod_{j=1}^{m} \frac{1}{1-j x}\right]=\frac{1}{m !} \sum_{k=0}^{m}(-1)^{m-k}\left(\begin{array}{c}
m \\
k
\end{array}\right) k^{n}
$$

being the Stirling number of the second kind (that is, the number of ways of partitioning a set of $n$ elements into $m$ non-empty subsets). The right-hand side of (A3) reduces to -1 at $u=-1$, proving part (ii). For part (iii), we use the residue theorem and the substitution $e^{t}=1+x$ to get

$$
\frac{1}{(n-1) !} \mathbf{C}_{u^{m}}\left[P_{n}(u)\right]=\operatorname{Res}_{t=0}\left[\frac{e^{t}\left(e^{t}-1\right)^{m-1} d t}{t^{n}}\right]=\operatorname{Res}_{x=0}\left[\frac{x^{m-1} d x}{[\log (1+x)]^{n}}\right]
$$

for $m, n \geqslant 1$.

We can now prove the equivalence of the two sets of relations described above. Let $R$ be the $\mathbb{Q}$-subalgebra of the Chow algebra generated (with respect to the Pontryagin product, which we 


\section{CyCle RELATIONS ON JACOBIAN VARIETIES}

denote simply by juxtaposition) by the $C_{(j)}$. We define two polynomials $G(t) \in R[t]$ and $H(u, t) \in$ $R[u, t]$ by

$$
G(t)=\sum_{a=0}^{g-1}(a+1) ! C_{(a)} t^{a+2}, \quad H(u, t)=\sum_{a=0}^{g-1} P_{a+2}(u) C_{(a)} t^{a+2} .
$$

Then the relations obtained from Theorem 1.1 can be written in the form

$$
\operatorname{deg}_{t}\left[G(t)^{s}\right] \leqslant d-r+s \text { for } 1 \leqslant s \leqslant r,
$$

while, in view of (A1), Herbaut's relations can be written in the form

$$
\mathbf{C}_{u^{d-r+s}}\left[\frac{1}{1+u} H(u, t)^{s}\right]=0 \quad \text { in } R[t], \quad \text { for } 1 \leqslant s \leqslant r .
$$

We also introduce the strengthened Herbaut relations

$$
\operatorname{deg}_{u}\left[H(u, t)^{s}\right] \leqslant d-r+s \text { for } 1 \leqslant s \leqslant r .
$$

Clearly (A6) implies (A5). (Note that $H(u, t)^{s} /(1+u)$ is a polynomial by part (ii) of the Lemma A.1.) To see that (A4) implies (A6), we use (A2) to obtain

$$
G\left(\frac{t}{\log (1+x)}\right)=H\left(\frac{1}{x}, t\right)-\varepsilon(x, t)
$$

with $\varepsilon(x, t)=\mathrm{O}(x)$ (in fact, $\left.\varepsilon(x, t)=\mathrm{O}\left(x t^{2}\right)\right)$ and, hence, assuming (A4),

$$
\begin{aligned}
H\left(\frac{1}{x}, t\right)^{s} & =\sum_{s^{\prime}=0}^{s}\left(\begin{array}{c}
s \\
s^{\prime}
\end{array}\right) G\left(\frac{t}{\log (1+x)}\right)^{s^{\prime}} \varepsilon(x, t)^{s-s^{\prime}} \\
& =\sum_{s^{\prime}=0}^{s} \mathrm{O}\left(\frac{1}{x^{d-r+s^{\prime}}}\right) \mathrm{O}\left(x^{s-s^{\prime}}\right)=\mathrm{O}\left(\frac{1}{x^{d-r+s}}\right)
\end{aligned}
$$

as $x \rightarrow 0$, proving (A6). To prove that (A5) implies (A4), we use induction on $s$. Assume (A5) for some $s \leqslant r$ and (A4) for all $s^{\prime}<s$. Equation (A7) and the inductive assumption give

$$
G\left(\frac{t}{\log (1+x)}\right)^{s}=H\left(\frac{1}{x}, t\right)^{s}+\mathrm{O}\left(\frac{1}{x^{d-r+s-2}}\right)
$$

and, hence, for $n>d-r+s-2$,

$$
\mathbf{C}_{t^{n}}\left[G(t)^{s}\right] \cdot \mathbf{C}_{x^{-d+r-s}}\left[\frac{x}{1+x} \frac{1}{[\log (1+x)]^{n}}\right]=\mathbf{C}_{t^{n}}\left[\mathbf{C}_{u^{d-r+s}}\left[\frac{1}{1+u} H(u, t)^{s}\right]\right]=0
$$

by (A5). Differentiating (A2) and using part (i) of Lemma A.1, we find that the second factor on the left equals $((d-r+s) ! /(n-1) !) \mathfrak{S}_{n-1}^{(d-r+s)}$, which is non-zero for $n>d-r+s$. Equation (A4) follows.

\section{REFERENCES}

Bea86 A. Beauville, Sur l'anneau de Chow d'une variété abélienne, Math. Ann. 273 (1986), 647-651.

Bea04 A. Beauville, Algebraic cycles on Jacobian varieties, Compositio Math. 140 (2004), 683-688.

CV93 E. Colombo and B. van Geemen, Notes on curves in a Jacobian, Compositio Math. 88 (1993), 333-353.

Ful98 W. Fulton, Intersection Theory, Ergebnisse der Mathematik und ihrer Grenzgebeite (3), vol. 2 (Springer, Berlin, 1998).

Her07 F. Herbaut, Algebraic cycles on the Jacobian of a curve with a linear system of given dimension, Compositio Math. 143 (2007), 883-899. 


\section{CyCle RELATIONS ON JACOBIAN VARIETIES}

Mac95 I. G. MacDonald, Symmetric functions and Hall polynomials, second edition, Oxford Mathematical Monographs (Clarendon Press, Oxford, 1995).

Mar05 G. Marini, Tautological cycles on Jacobian varieties, Preprint (2005), arXiv:math/0509659.

Gerard van der Geer geer@science.uva.nl

Korteweg-de Vries Instituut, Universiteit van Amsterdam, Plantage Muidergracht 24, 1018 TV

Amsterdam, The Netherlands

Alexis Kouvidakis kouvid@math.uoc.gr

Department of Mathematics, University of Crete, GR-71409 Heraklion, Greece

Don Zagier zagier@mpim-bonn.mpg.de

Max-Planck-Institut für Mathematik, Vivatsgasse 7, 53111 Bonn, Germany 